\title{
Original
}

\section{Comparison of the Morphological Structures of the Human Calvarium and Turtle Shell}

\author{
Noboru Ishikawa ${ }^{1)}$, Yuzo Hirayama ${ }^{1)}$, Yasuo Miake ${ }^{1)}$, Kei Kitamura ${ }^{1)}$, Norio Kasahara ${ }^{2)}$, Shinichi Abe ${ }^{3)}$ and Hitoshi Yamamoto ${ }^{1)}$ \\ 1) Department of Histology and Developmental Biology, Tokyo Dental College, Tokyo, Japan \\ 2) Department of Forensic Odontology and Anthropology, Tokyo Dental College, Tokyo, Japan \\ 3) Department of Anatomy, Tokyo Dental College, Tokyo, Japan \\ (Accepted for publication, July 12, 2019)
}

\begin{abstract}
In the field of forensic odontology, not only personal identification using oral conditions, including dental treatment marks and DNA typing, but also species identification, age or sex estimation using cranial or partial bones, and time after death may also be applicable as estimation methods. Among these many tasks, one of the most difficult is species identification of fragmented calvarium. This is because the calvarium has poor morphological features, except that it is a flat bone, and few reports have described how to differentiate it. In this study, a simple identification method for turtle shell (carapace and plastron) whose morphological characteristics closely resembled those of the calvarium was applied. As a result, in an enlarged image obtained using a stereoscopic microscope, the characteristics of each suture pattern could be confirmed. In the decalcified and non-decalcified bone specimens, the difference in the inner/outer laminar structure and the specific structural difference of the cancellous bone-equivalent part were confirmed. Furthermore, most of the features could be obtained in the destructive inspection when it was examined using micro CT imaging to determine whether discrimination by nondestructive inspection was possible or not. No significant difference in ingredients was found using the Electron Probe X-ray Micro Analyzer. The calvarium was more calcified than the carapace but less calcified than the plastron. From these results, we suggest that micro CT imaging is effective for discriminating between the calvarium and turtle shell in a short time.
\end{abstract}

Key words: Forensic odontology, Calvarium, Turtle shell, Species identification, Computed tomography

\section{Introduction}

The main subjects of forensic odontology are cranial bones. These are used not only for personal identification using oral characteristics, including dental treatment marks and DNA typing, but also for species identification and age and sex estimation. When making an expert appraisal of a specimen using anthropological methods, it must first be determined whether the specimen is a bone and whether it is of human origin. Long and facial bones are relatively easy to identify because they have many distinct features ${ }^{1,2)}$. However, the calvarium, which is a flat bone, is often found as a partial bone, due to its many sutures, and species identification is often difficult, due to its lack of morphological features. One of the living things that makes species identification difficult is the turtle, which exists close to the human living environment. The turtle shell has a flat shape like the calvarium, so fragments of the calvarium are very similar to the turtle shell, making identification particularly difficult ${ }^{3}$. Many studies on the development and origin of turtle hard tissues have been reported from $>100$ years ago, including Odontochelys from around 220 million years ago, Pappochelys from around 240 million years ago, and reptiles from around 260 million years ago. Several origin theories such as Eunotosaurus have been reported, but the whole story has not yet been clarified ${ }^{4-10)}$. The turtle shell consists of the carapace (shell of the back) and the plastron (shell of the abdomen). The

Correspondence to: Dr. Noboru Ishikawa, Department of Histology and Developmental Biology, Tokyo Dental College, 2-9-18 Kanda-Misakicho, Chiyoda-ku, Tokyo, 101-0061 Japan; Tel: +81-3-6380-9274; E-mail: ishikawanoboru@tdc.ac.jp carapace is formed by ribs spreading out from the vertebral bodies. These ribs touch each other and form sutures, forming the carapace as a mass. The plastron is also thought to be formed by abutting ribs, similarly to the carapace ${ }^{11-16)}$. These sutures, much like in the calvarium, exhibit a serrated structure, and fibrous tissue enters the gaps. Therefore, the shell is difficult to differentiate from fragmented human bones because the sutures may dissociate with the decomposition of soft tissue after death. In this study, comparison of structures and quantitative analysis of components were performed on decalcified and non-decalcified bone specimens using human cranial bones and turtle shell (carapace and plastron). In addition, micro computed tomography (CT) images were used to determine whether identification by non-destructive inspection is possible.

\section{Materials and Methods}

Samples

Three donated calvaria of cadavers ( 2 males and 1 female; age at death, 54-88 years) were examined. All the cadavers were donated to the Tokyo Dental College for research and education on human anatomy and were fixed with arterial perfusion of $10 \% v / v$ formalin solution and stored in $50 \% v / v$ ethanol solution for $>3$ months. The turtle sample used was from the red-eared slider Trachemys scripta elegans (carapace: $16 \times 13 \mathrm{~cm}$, plastron: $15 \times 10 \mathrm{~cm}$; Fig. 1).

\section{Specimen Preparation}

First, the surface texture and shape of the suture patterns were ob- 


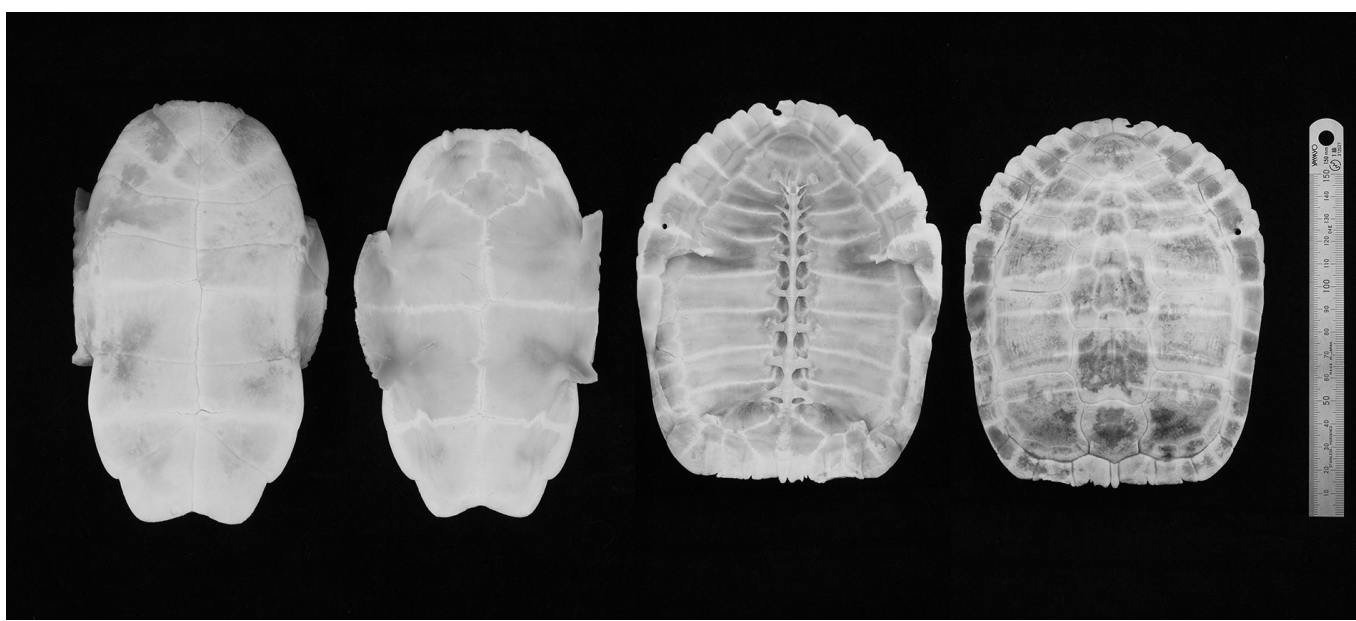

Figure 1. Photographs of the entire turtle shell (from the right: carapace-outside, carapace-inside, plastron-inside, and plastron-outside)

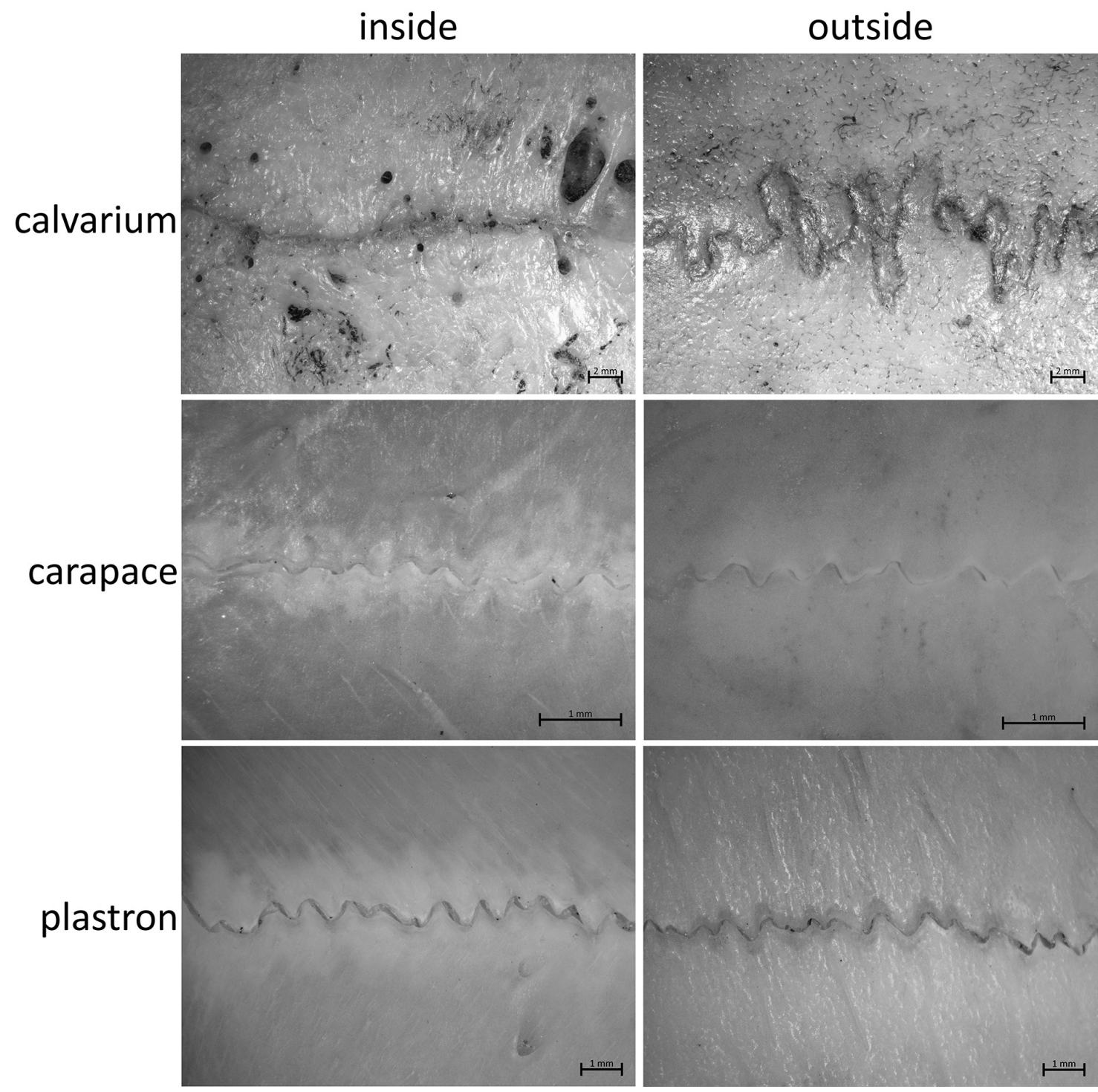

Figure 2. Stereoscopic microscope image of the morphological structure of the surface texture and the shape of the suture. The calvarium shows an irregular serrated structure, whereas the shell shows a somewhat regular and narrow structure. The surface texture shows lubricating properties inside the carapace and plastron as compared with inside/outside the calvarium and outside the carapace and plastron (scale bar: top, $2 \mathrm{~mm}$; middle and low, $1 \mathrm{~mm}$ ). 

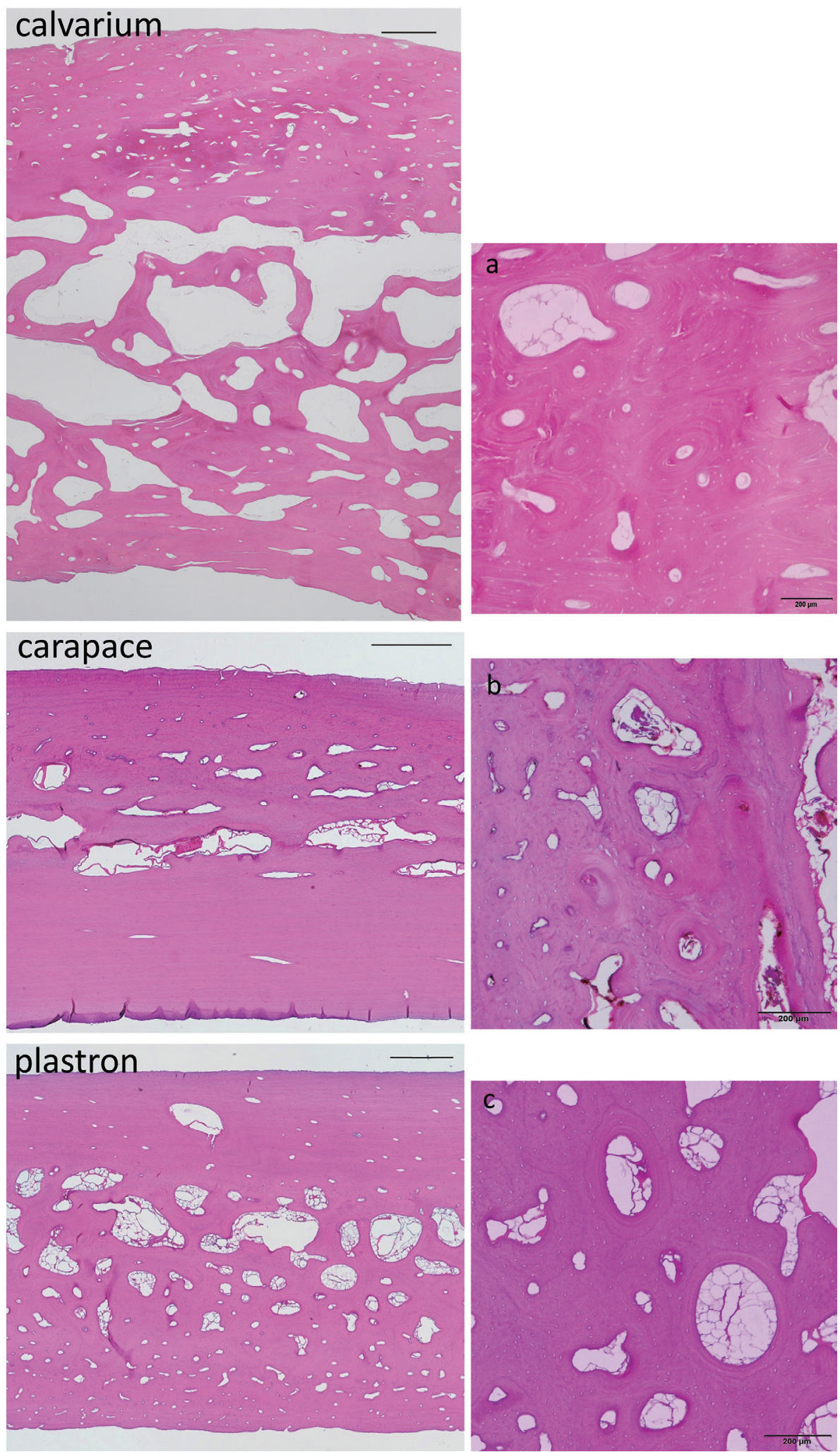

Figure 3. Decalcified bone specimens of three hard tissues (hematoxylin-eosin -stained images). Top left: whole image of the calvarium; middle left: whole image of the carapace; bottom left: whole image of the plastron (scale bar: $600 \mu \mathrm{m}$ ); top right (a): enlarged image of the osteon in the calvarium; middle right (b): enlarged image of the osteon in the carapace; bottom right (c): enlarged image of the osteon in the plastron (scale bar: $200 \mu \mathrm{m})$. Bone lacunae observed in the three hard tissues. The lamellar structure of the carapace and plastron inside/outside are different from the layered plate structure of the calvarium. In addition, the carapace and plastron had narrower cancellous bone spaces than the calvarium and showed no human-like cancellous bone or osteon-like structures with a large lumen. 


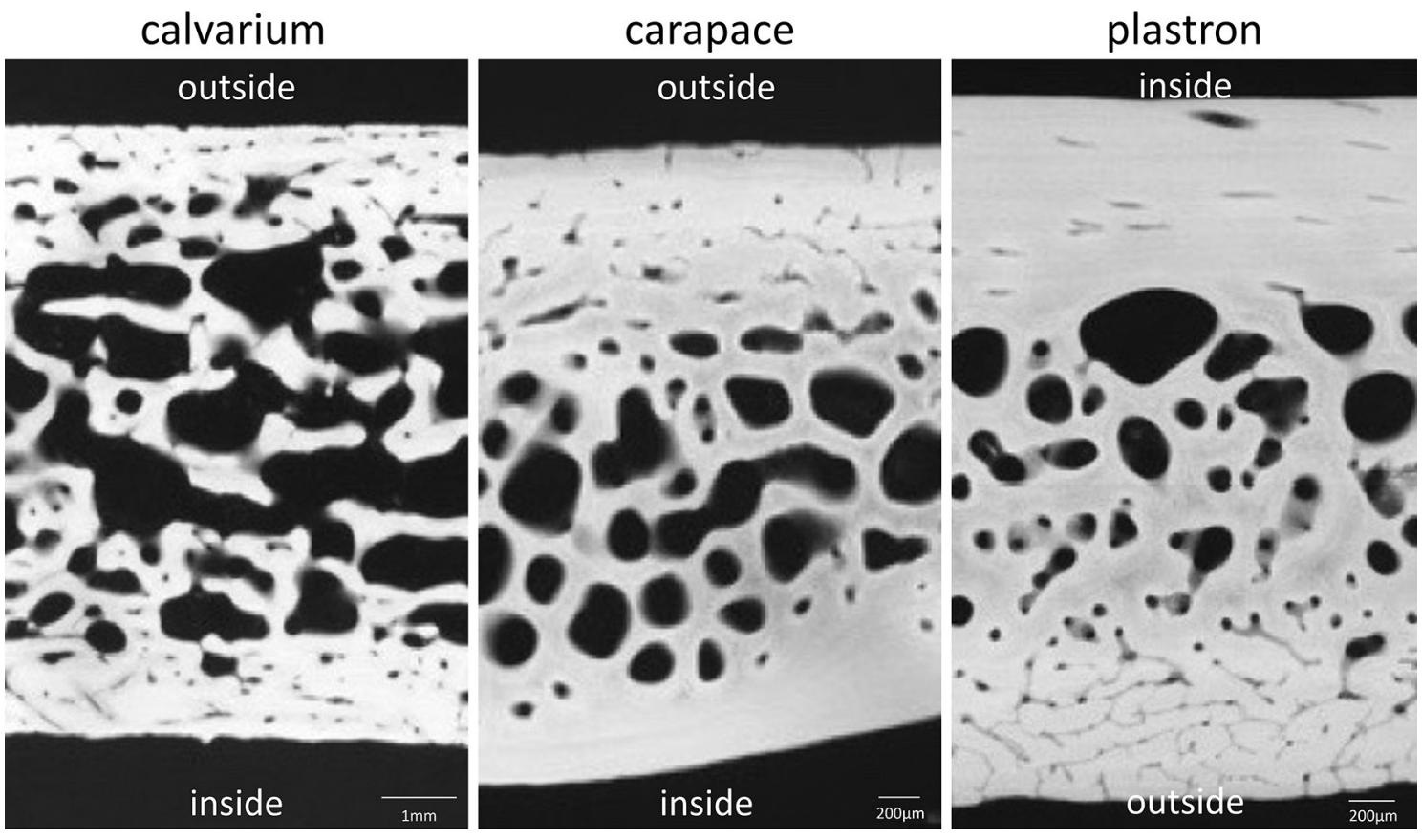

Figure 4. Contact micro-radiography of the three hard tissues. The inside/outside of the calvarium shows a similar structure, whereas the inside of the turtle shell has a tight bone matrix structure and is rough on the outside. Moreover, the cancellous bone space is wide in the calvarium and narrow in the turtle shell (scale bar: left, $1 \mathrm{~mm}$; middle and right, $200 \mu \mathrm{m}$ ).
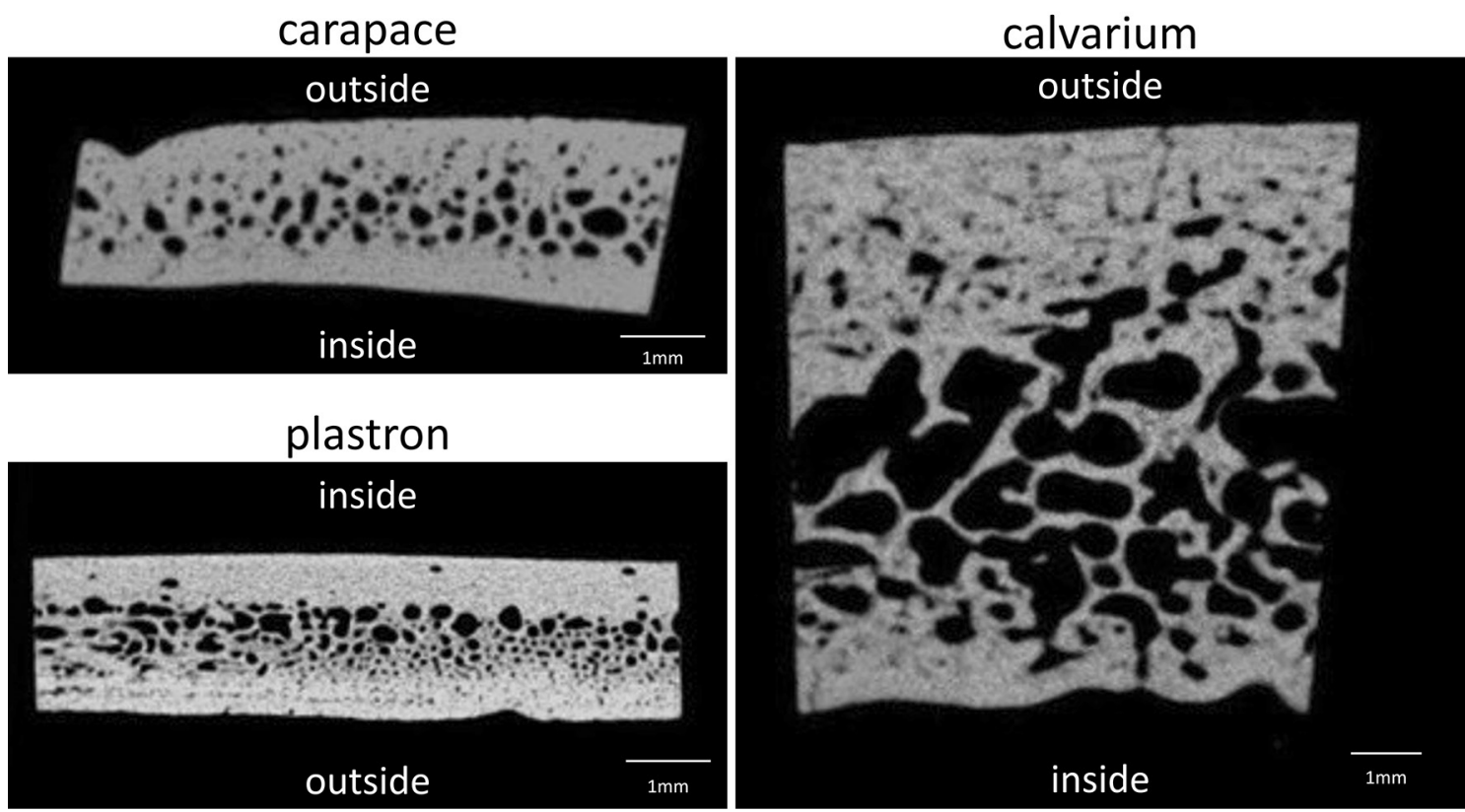

Figure 5. Micro computed tomography images of the three hard tissues. In addition to its ability to observe differences between the inside/outside lamellar structures, contact micro-radiography can detect characteristics of the cancellous bone equivalent (scale bar: 1 $\mathrm{mm})$.

served using a stereoscopic microscope (Stemi 508, Carl Zeiss Co., Ltd., Germany) with Axio Cam ERc 5S (Carl Zeiss Co., Ltd., Germany) and ZEN blue edition (Carl Zeiss Co., Ltd., Germany). Then, K-CX (Falma, Japan) was used for decalcification to prepare the decalcified bone specimens. Hematoxylin-eosin (H\&E)-stained specimens were prepared according to the conventional method. For preparation of ground sections from non-decalcified bone specimens, embedding resin No. 105 (Marumoto Struers, Japan) was used to embed the samples. Thereafter, the samples were polished to a thickness of $120 \mathrm{~mm}$ and photographed with contact micro-radiography (CMR) using a soft X-ray generator (Softex CMR-3, Softex Co, Japan), with a tube voltage of $15 \mathrm{kV}$, tube current of $3 \mathrm{~mA}$, and photography time of 6 minutes. A high-precision photo plate (Konica Minolta, Inc., Japan) was used for the X-ray film. After photography, the film was developed with a Kodak D-19 developer at $20^{\circ} \mathrm{C}$ for 5 minutes. Then, the film was fixed for 5 minutes using Super Fujifix-L (Fujifilm Holdings Corp., Japan), washed with water, and dried. 


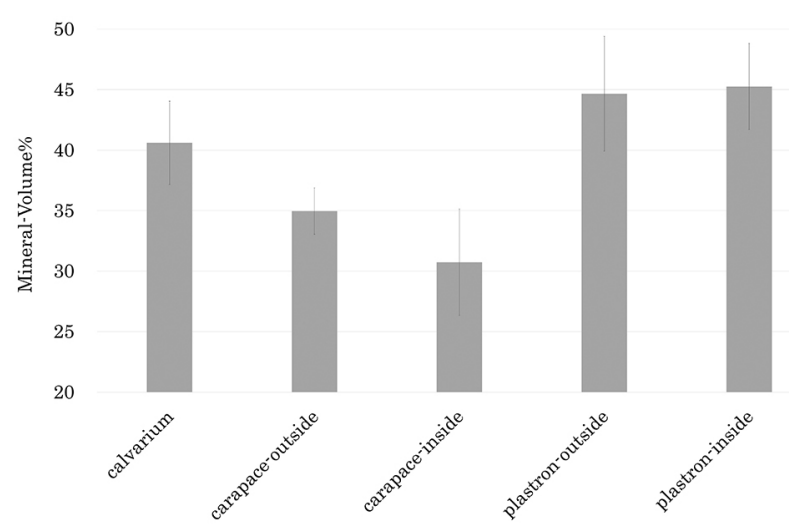

Figure 6. Comparison of the degree of calcification of the three hard tissues ( 5 sites). The degree of calcification of the calvarium was higher than that of the carapace and lower than that of the plastron. The highest degree of calcification was found in the plastron, and the lowest degree of calcification was found in carapace-inside. Highly significant difference was observed except between outside and inside the plastron $(\mathrm{p}<0.01)$.
After that, for comparison of the degree of calcification, the image was taken directly from a CMR image and entered into a computer. Digital imaging was performed with an 8-bit grayscale using an image analysis software (Image-Pro PLUS, Nippon Roper K.K.), and gray values were calculated. The gray value was determined in an area of $50 \mu \mathrm{m}$ in width and $50 \mu \mathrm{m}$ in height ( 1 site). After the total 30 sites at 3 samples (10 sites per sample) were measured, each gray value was converted to the aluminum equivalent, according to the calibration curve of the aluminum step wedge. The mineral-volume $\%$ was calculated using the method described by Angmar et $\mathrm{al}^{17}$. In addition, micro CT imaging (tube voltage: $140 \mathrm{kV}$, tube current: $224 \mathrm{~mA}$ ) was performed with the Microfocus X-ray RTR/CT System (Tesco Corp., Japan) to determine whether the two species could be distinguished by non-destructive inspection. Finally, to compare the elemental composition of the calvarium, carapace, and plastron, vapor deposition was performed using a Carbon Coater (VC-100S, Vacuum Device, Japan). For quantitative analysis of the bone surface, 30 points (10 points per sample) were measured using the Electron Probe X-ray Micro Analyzer (EPMA; JXA-8200, JEOL Ltd., Japan).
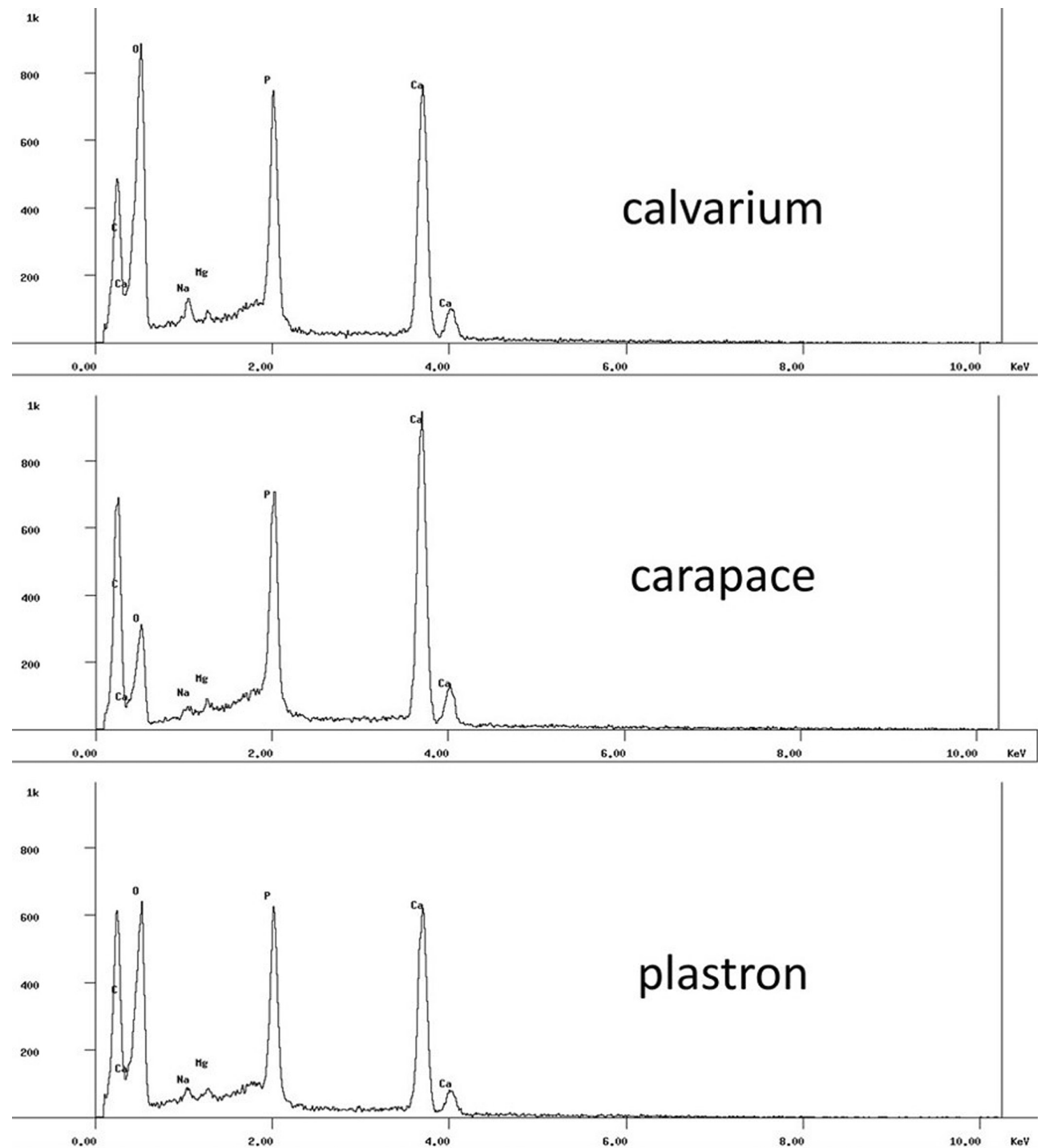

Figure 7. Analysis of the components of the three hard tissues using an Electron Probe X-ray Micro Analyzer. C, Ca, O, Na, Mg, and P were detected in all three hard tissues. 


\section{Ethical Approval}

The medical and ethical committees of Tokyo Dental College approved all the human samples and research protocols used in this study (Approval No. 843).

\section{Statistical Analysis}

Statistical analysis was performed using the Kruskal-Wallis test. Regression analysis was conducted using Microsoft Excel software and Stat Plus LE.

\section{Results}

\section{Morphological Comparison using Stereoscopic Microscopy}

In the calvarium, the foramen nutricium could be observed on the bone surface with the naked eye, whereas there were no macroscopic features observed on the carapace and plastron. The calvarium displayed an irregular serrated structure at the sutures, whereas the carapace and plastron had a narrow width and a comparatively regular serrated structure. In addition, the sutures of the plastron showed a somewhat fragile binding pattern compared with the sutures of the carapace (Fig. 2).

\section{Morphological Comparison of Hematoxylin-Eosin-stained Tissue Specimens}

The calvarium, carapace, and plastron all clearly showed numerous bone lacunae in the matrix. In the carapace, a thick laminar structure was observed, with the inner layer thicker than the outer layer and a rough outer structure in contrast to the inner structure. Similarly, there was a thick laminar structure inside the plastron, and a rough outer structure was noted. The carapace and plastron also had a narrower trabecular gap in the cancellous tissue than the calvarium. Moreover, the lamellar structure of the carapace and plastron inside/outside showed differences from the layered plate structure of the calvarium. In addition, the carapace and plastron had narrower cancellous bone spaces than the calvarium and showed osteon-like structures with large lamina that was different from that of the human-like cancellous bone structures. The calvarium, carapace, and plastron all had Haversian canals and osteon-like structures, but these structures were less obvious in the carapace and plastron (Fig. 3).

\section{Morphological Comparison of Contact Micro-Radiography and Micro CT Images}

In the CMR and micro CT images, as in the case of $\mathrm{H} \&$ E-stained images, thick lamellar structures like inside of the carapace and plastron were not observed in the calvarium, and the structure of the cancellous bone was also clearly observed. The bone lacunae, which could be observed in H\&E-stained images of the matrix, could not be observed in CMR and micro CT images. Many Volkmann-canal-like structures, similar to human compact bone, were observed on the outside of the carapace and plastron. Comparison of the inner and outer structures showed the bone matrix structure and a smooth surface texture. The characteristics of the trabecular gap observed in the H\&E-stained images were also confirmed by the micro CT and CMR images. The micro CT and CMR images mostly confirmed similar findings (Figs. 4 and 5).

\section{Comparison of the Degree of Calcification using Contact Micro-Radi- ography}

The comparison of the degrees of five measurement sites (Group 1: calvarium, Group 2: carapace-outside, Group 3: carapace-inside, Group 4: plastron-outside, and Group 5: plastron-inside) revealed significantly different degrees of calcification except between plastron-outside and plastron-inside $(\mathrm{p}<0.01)$.

In addition, the plastron had a higher degree of calcification than the carapace. Plastron had the highest degree of calcification, and carapace-inside had the lowest degree of calcification (Fig. 6).

\section{Composition Analysis using the Electron Probe X-ray Micro Analyzer}

The qualitative analysis of the three hard tissues (calvarium, carapace, plastron) revealed $\mathrm{C}, \mathrm{Ca}, \mathrm{O}, \mathrm{Na}, \mathrm{Mg}$, and $\mathrm{P}$ in all the sites (Fig. 7). As the results of the quantitative analysis of five measurement sites (Group 1: calvarium, Group 2: carapace-outside, Group 3: carapace-inside, Group 4: plastron-outside, and Group 5: plastron-inside) showed significant differences, but no element exhibited characteristic content in each hard tissue.

\section{Discussion}

The field of forensic odontology involves personal identification of unknown corpses using oral characteristics, including dental treatment marks. It also involves a wide range of tasks such as sex and age estimation using skeletal remains and DNA typing, as well as estimation of time since death ${ }^{18-25)}$. Most subjects are appraised using cranial bones, including the calvarium. The calvarium is appraised primarily for species identification, but its morphological features are poor, except that it is a flat bone, and few reports have described its appraisal method. In this study, we observed differences in bone surface texture and suture morphology between calvarium and turtle shell using stereoscopic microscopy. However, it may be difficult to identify species only using suture patterns of the calvarium because the closure of sutures is generally considered an age-related change and individual differences in surface texture and width of the calvarium have been noted ${ }^{26,27}$. Furthermore, compared with the carapace, which has tightly fitted sutures, it is thought that the plastron, in which the suture gaps are wider, is more likely to separate after death. Therefore, the discovery of a partial plastron is highly likely. Although differences in suture width and morphology were observed between calvarium and turtle shell in the present study, detached bone fragments are often found with their edges worn due to external physical force, wherein the differentiation between calvarium and turtle shell based solely on these findings may not be effective. However, for cases in which sutures remain intact in the discovered bone fragments, the narrow and regular suture morphology of turtles will likely be a useful feature to look for during identification. In H\&Estained images, clear differences between the calvarium and turtle shell could be confirmed in compact and cancellous bones, and the structural features inside/outside were clearly visible. The thicker inner lamellar structure observed in turtle shell and the differences in the outer structure, which is distinct from the inner structure, and in the medullary cavity structure in cancellous bones compared to calvarium are considered to have evolved for effective distribution of external physical forces that may be experienced in nature and for protection from predators ${ }^{28,29}$. Furthermore, the morphological characteristics of each hard tissue necessary for identification could be confirmed using micro CT imaging, which is a non-destructive inspection, and CMR. Hence, micro CT imaging is suggested as an easy and fast non-destructive method to distinguish between calvarium and turtle shell. In addition, quantitative analysis of each constituent element revealed the same components in the carapace, plastron, and calvarium, with no notable differences in the amounts of contained elements. This confirmed that turtle shell has almost the same composition as human bone. In addition, the degree of calcification of the calvarium was higher than that of the carapace and 
lower than that of the plastron. As the degree of calcification was significantly different between the carapace and plastron, this might be useful for their identification. However, comparison of the degree of calcification combined with tissue structure analysis is the most effective method of identification, as differences in the number and size of lumina present affect the calcification measurement values. Taken together, the findings demonstrated that differential identification between calvarium and turtle shell based solely on differences in suture morphology and composition is difficult. Instead, it was shown that findings such as the thick inner lamellar structure, the rough outer structure that is distinct from the inner structure, and the narrow medullary cavity of cancellous bones, which are characteristics of a turtle shell, as observed on non-destructive micro CT imaging, allow quick and easy differentiation. Additionally, the differences in the degree of calcification observed in the present study were significant and may be used as an effective identification parameter. However, further investigation is required in terms of variation in the degree of calcification using individuals of different body sizes, considering the long lifespan of turtles.

In this study, micro CT imaging was effective as an identification method. Therefore, we suggest that micro CT is useful for easily and quickly distinguishing turtle shell from the calvarium.

\section{Conflict of Interest}

The authors have declared that no conflict of interest exists.

\section{References}

1. Blau S and Briggs CA. The role of forensic anthropology in Disaster Victim Identification (DVI), Forensic Sci Int 25: 29-35, 2011

2. Rérolle C, Saint-Martin P, Dedouit F, Rousseau H and Telmon N. Is the corticomedullary index valid to distinguish human from nonhuman bones: a multislice computed tomography study. Forensic Sci Int 10: e1-5, 2013

3. Diane L France. Human and Nonhuman Bone Identification A Color Atlas. CRC Press, pp. 25, 2009

4. Li C, Wu XC, Rieppel O, Wang LT and Zhao LJ. An ancestral turtle from the Late Triassic of southwestern China. Nature 27: 497-501, 2008

5. Bever GS, Lyson TR, Field DJ and Bhullar BS. The amniote temporal roof and the diapsid origin of the turtle skull. Zoology 119(6): 471-473, 2016

6. Lyson TR, Bever GS, Scheyer TM, Hsiang AY and Gauthier JA. Evolutionary origin of the turtle shell. Curr Biol 17: 1113-1119, 2013

7. Joyce WG. The origin of turtles: a paleontological perspective. J Exp Zool B Mol Dev Evol 324(3): 181-193, 2015

8. Bever GS, Lyson TR, Field DJ and Bhullar BA. Evolutionary origin of the turtle skull. Nature 10: 239-242, 2015

9. Schoch RR and Sues HD. The diapsid origin of turtles. Zoology 119(3): 159-161, 2016

10. Schoch RR and Sues HD. A new stem-turtle from the Middle Triassic of Germany and the evolution of the turtle body plan. Nature 30 : 584-587, 2015

11. Hirasawa T, Nagashima $H$ and Kuratani $S$. The endoskeletal origin of the turtle carapace. Nat Commun 4: 2107, 2013

12. Lyson TR, Bhullar BA, Bever GS, Joyce WG, de Queiroz K, Abzhanov A and Gauthier JA. Homology of the enigmatic nuchal bone reveals novel reorganization of the shoulder girdle in the evolution of the turtle shell. Evol Dev 15(5): 317-325, 2013

13. Nagashima H, Shibata M, Taniguchi M, Ueno S, Kamezaki N and Sato N. Comparative study of the shell development of hard- and soft-shelled turtles. J Anat 225(1): 60-70, 2014

14. Cordero GA and Quinteros K. Skeletal remodelling suggests the turtle's shell is not an evolutionary straitjacket. Biol Lett 2015: doi: 10.1098/rsbl.2015.0022.

15. Scheyer TM, Brüllmann B and Sánchez-Villagra MR. The ontogeny of the shell in side-necked turtles, with emphasis on the homologies of costal and neural bones. J Morphol 269(8): 1008-1021, 2008

16. Moustakas-Verho JE, Cebra-Thomas J and Gilbert SF. Patterning of the turtle shell. Curr Opin Genet Dev 45: 124-131, 2017

17. Angmar B, Carlstrom D and Glas JE. Studies on the ultrastructure of dental enamel. IV. The mineralization of normal human enamel. J Ultrastruct Res 8: 12-23, 1953

18. Marroquin TY, Karkhanis S, Kvaal SI, Vasudavan S, Kruger E and Tennant M. Age estimation in adults by dental imaging assessment systematic review. Forensic Sci Int 275: 201-211, 2017

19. Khorate MM, Dinkar AD and Ahmed J. Accuracy of age estimation methods from orthopantomograph in forensic odontology: a comparative study. Forensic Sci Int 234: e1-8, 2015

20. Karkhanis S, Mack P and Franklin D. Dental age estimation standards for a Western Australian population. Forensic Sci Int 257: e1-9, 2015

21. Capitaneanu C, Willems G, Jacobs R, Fieuws S and Thevissen P. Sex estimation based on tooth measurements using panoramic radiographs. Int J Legal Med 131(3): 813-821, 2017

22. Silva AM, Pereira ML, Gouveia S, Tavares JN, Azevedo Á and Caldas IM. A new approach to sex estimation using the mandibular canine index. Med Sci Law 56(1): 7-12, 2016

23. Ishikawa $\mathrm{N}$, Miake $\mathrm{Y}$, Kitamura $\mathrm{K}$ and Yamamoto $\mathrm{H}$. A new method for estimating time since death by analysis of substances deposited on the surface of dental enamel in a body immersed in seawater. Int J Legal Med 2019: doi: 10.1007/s00414-019-02020-5

24. Corrêa HSD, Pedro FLM, Volpato LER, Pereira TM, Siebert Filho $\mathrm{G}$ and Borges ÁH. Forensic DNA typing from teeth using demineralized root tips. Forensic Sci Int 280: 164-168, 2017

25. Minaguchi K, Maruyama S, Kasahara I, Nohira C, Hanaoka Y, Tsai T, Kiriyama $\mathrm{H}$ and Takahashi N. Identification of unknown body using DNA analysis and dental characteristics in chest X-ray photograph. Bull Tokyo Dent Coll 46(4): 145-153, 2005

26. Ishikawa N, Suganami H, Nishida A, Miyamori D, Kakiuchi Y, Yamada N, Wook-Cheol K, Kubo T and Ikegaya H. Utilization of bone impedance for age estimation in postmortem cases. J Forensic Leg Med 36: 102-107, 2015

27. De Boer HH, Van der Merwe AE and Soerdjbalie-Maikoe VV. Human cranial vault thickness in a contemporary sample of 1097 autopsy cases: relation to body weight, stature, age, sex and ancestry. Int J Legal Med 130(5): 1371-1377, 2016

28. Achrai B and Wagner HD. The turtle carapace as an optimized multi-scale biological composite armor - A review. J Mech Behav Biomed Mater 73: 50-67, 2017

29. An B and Wagner HD. Protection mechanisms of the carapace of a box turtle. J Mech Behav Biomed Mater 71: 54-67, 2017 
J.Hard Tissue Biology Vol. 28(3): 289-296, 2019 